\title{
AVALIAÇÃO DO CONTATO PNEU-SOLO EM TRÊS MODELOS DE PNEUS AGRÍCOLAS
}

\author{
FLÁVIO R. MAZETTO ${ }^{1}$, KLÉBER P. LANÇAS ${ }^{2}$, ALBERTO K. NAGAOKA ${ }^{3}$, \\ PEDRO CASTRO NETO ${ }^{4}$, SAULO P. S. GUERRA ${ }^{5}$
}

\begin{abstract}
RESUMO: Este trabalho teve como objetivo avaliar as áreas de contato, as deformações elásticas dos pneus, a resistência do solo à penetração e os perfis do recalque no solo de três modelos de rodados pneumáticos. O ensaio seguiu um delineamento experimental casualizado, constituído por 12 tratamentos e quatro repetições, nos quais se avaliaram os modelos de pneus de carcaça diagonal, radial e o terceiro de configuração mista, denominado BPAF, inflados com as pressões ideais e submetidos a cargas radiais de $5 ; 10 ; 15$ e $20 \mathrm{kN}$, simulando o que ocorre no campo. $\mathrm{O}$ ensaio dos pneus agrícolas foi realizado no Núcleo de Ensaio de Máquinas e Pneus Agrícolas - NEMPA, da Faculdade de Ciências Agronômicas - UNESP, Câmpus de Botucatu - SP, em uma prensa hidráulica sobre um tanque de solo. Os resultados relativos às áreas de contato e deformações elásticas mostraram valores maiores para o pneu BPAF. Os recalques do pneu BPAF no solo foram menores comparados aos outros rodados, e com os pneus radial e BPAF houve menores resistências do solo à penetração.
\end{abstract}

PALAVRAS-CHAVE: carga radial, resistência à penetração, recalque.

\section{EVALUATION OF FOOT PRINT FOR THREE AGRICULTURAL TIRE TYPES}

SUMMARY: The aim of this study was to evaluate the tire/ground contact areas, tire elastic deformation, cone index and foot printed on the ground of tire models. The experiment was done in 12 treatments and four repetitions with three tire models: bias ply, radial and bias belt. The tires were inflated with right inflation pressures and four radial loads were applied on the wheels: $5 ; 10 ; 15$ and $20 \mathrm{kN}$, simulating what happens in the field. The agricultural tire tests were carried out at Sao Paulo State University, Botucatu - SP, Brazil, using a hydraulic press over the soil bin. The contact area and elastic deformation results showed highest values for bias belt tire. The soil sinkage of bias belt tire was smaller than other pneumatic tires. The radial and bias belt tires exercised smaller cone index than bias ply tire.

KEYWORDS: radial load, cone index, sinkage.

\section{INTRODUÇÃO}

Na recente década, vários estudos e experimentos têm sido efetuados a fim de melhor conhecer as características dos pneus agrícolas, principalmente no estudo da interação pneu/solo que está relacionada com o desempenho dos tratores.

A utilização do trator numa propriedade é variada, devendo-se considerar que tipos de pneus e pressões de inflação inadequadas representam elementos negativos para a maior parte das operações culturais, induzindo a compactação do solo, a emissão de gases poluentes ao ambiente, bem como o aumento de consumo de energia durante a execução do trabalho (MAZETTO, 2004).

\footnotetext{
${ }^{1}$ Eng ${ }^{\text {o }}$ Agrônomo, aluno de doutorado do curso de Pós-Graduação em Agronomia (Energia na Agricultura), FCA/UNESP, Botucatu SP, Fone: (0XX14) 6802.7118, e-mail: frmazetto@fca.unesp.br

${ }^{2}$ Eng $^{\mathrm{o}}$ Mecânico, Professor Adjunto, Departamento de Engenharia Rural, FCA/UNESP, Botucatu - SP.

${ }^{3}$ Eng ${ }^{0}$ Agrícola, Professor Doutor, Departamento de Engenharia Rural, UDESC, Lages - SC.

${ }^{4}$ Eng ${ }^{\mathrm{O}}$ Agrônomo, Professor Titular, Departamento de Engenharia Rural, UFLA, Lavras - MG.

${ }^{5}$ Eng ${ }^{\mathrm{o}}$ Florestal, aluno de doutorado do curso de Pós-Graduação em Agronomia (Energia na Agricultura), FCA/UNESP, Botucatu - SP. Recebido pelo Conselho Editorial em: 2-7-2003

Aprovado pelo Conselho Editorial em: 26-7-2004
} 
As características de desempenho de pneus agrícolas em diferentes condições de solo, com várias cargas dinâmicas e pressões de inflação, são de grande interesse na adequação dos mesmos dentro da unidade de força motriz, tendo como objetivo a execução dos trabalhos de campo de forma mais eficiente (UPADHYAYA \& WULFSOHN, 1990).

SHARMA \& PANDEY (1996) afirmaram que as características de eficiência de um rodado dependem, em grande parte, não somente da pressão de contato, mas também do tamanho e da forma da área de contato, concluindo que, para um rodado pneumático, a aproximação elíptica é o melhor estimador para a área de contato pneu-superfície.

PORTERFIELD \& CARPENTER (1986), utilizando a pressão média de contato de um pneu em uma superfície rígida, encontraram que o nível de compactação superficial depende da pressão de contato, enquanto a compactação profunda depende da área de contato, largura do pneu e da carga suportada pelo mesmo.

Assim, o presente trabalho teve como objetivo avaliar e comparar as áreas de contato, deformações elásticas dos pneus, resistência à penetração do solo e os perfis do recalque no solo de diferentes modelos de rodados - radial, diagonal e BPAF (baixa pressão e alta flutuação) - com pressões de inflação ideais e combinando quatro condições de lastragem dos pneus $(5 ; 10 ; 15$ e $20 \mathrm{kN})$.

\section{MATERIAL E MÉTODOS}

A presente pesquisa foi realizada no Núcleo de Ensaio de Máquinas e Pneus Agrícolas (NEMPA), do Departamento de Engenharia Rural da Faculdade de Ciências Agronômicas - UNESP, Câmpus de Botucatu - SP.

Para a determinação da área de contato de um pneu agrícola, foi utilizada uma prensa hidráulica constituída de perfis e tubos de aço-carbono, acionada por meio de um pistão hidráulico. Sob a prensa, um carro móvel sustentava um tanque de solo, apoiado sobre rodas de aço, as quais deslizavam sobre trilhos (Figura 1).

O tanque de solo, preenchido com uma amostra de Latossolo Vermelho, textura média, apresentando teor de água de $0,0804 \mathrm{~kg} \mathrm{~kg}^{-1}(8,04 \%)$, foi dividido em quatro camadas de $0,10 \mathrm{~m}$, sendo três delas preenchidas com $250 \mathrm{~kg}$ de solo e a última com $230 \mathrm{~kg}$. Após o preenchimento de cada camada do tanque, foi utilizado um rolo compactador para nivelar e compactar o solo, fazendo com que o volume do tanque de $741 \mathrm{dm}^{3}$ fosse preenchido com $980 \mathrm{~kg}$ da amostra de solo, obtendo uma densidade final de $1,32 \mathrm{~kg} \mathrm{dm}^{-3}$.

Três modelos de pneus existentes no mercado foram ensaiados, um dos quais com carcaça diagonal (14.9-26) de fabricação nacional, outro com carcaça radial (14.9R26) e o terceiro de configuração mista (500/60-26.5), denominado pneu de baixa pressão e alta flutuação (BPAF), ambos importados (Figura 2).

Os pneus agrícolas foram inflados com as pressões recomendadas por GOODYEAR (1994) e TRELLEBORG (2002) para cada tipo de pneu, tomando-se como base a pressão de inflação para a carga radial máxima, ou seja, 103,43 kPa (15 psi) para o pneu BPAF; $186,17 \mathrm{kPa}$ (27 psi) para o pneu radial e $206,85 \mathrm{kPa}$ (30 psi) para o pneu diagonal.

As cargas radiais foram atribuídas aos pneus de forma crescente e gradativa, sendo a carga mínima de $5 \mathrm{kN}$ e os carregamentos subseqüentes de 10; 15 e $20 \mathrm{kN}$.

O pneu foi fixado em um eixo da prensa hidráulica e, segundo os procedimentos de carregamentos adotados, realizou-se a prensagem do rodado agrícola sobre o solo, obtendo a estampa da banda de rodagem do pneu e seu recalque sobre a superfície deformável. 


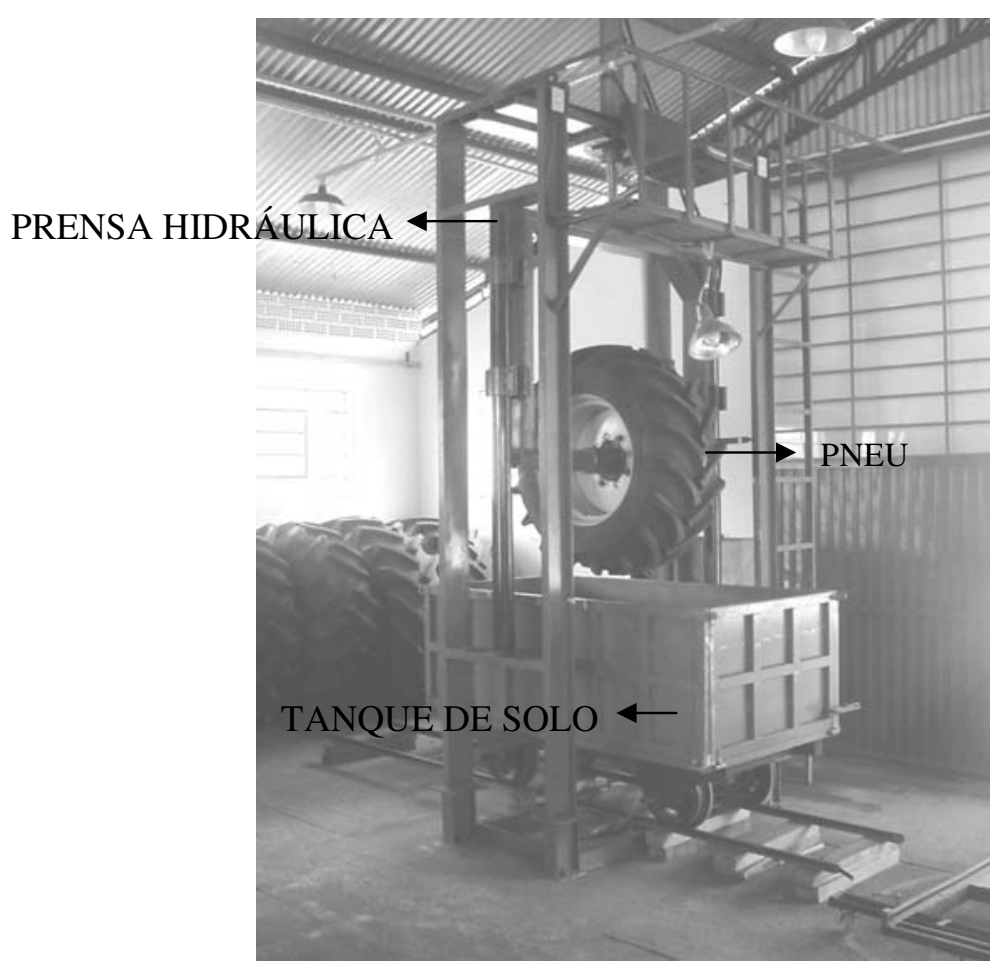

FIGURA 1. Prensa hidráulica e tanque de solo.

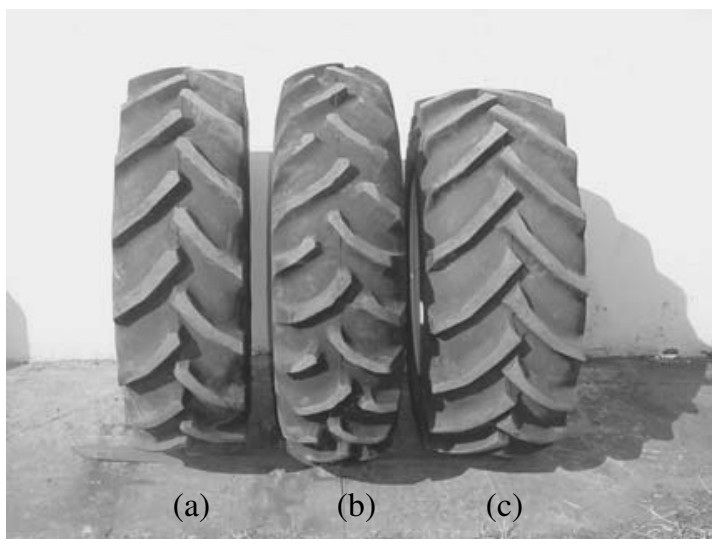

FIGURA 2. Pneus ensaiados: radial (a), diagonal (b) e BPAF (c), respectivamente.

Após cada prensagem, um quadro de madeira foi colocado e ajustado sobre o solo para ser fotografado junto com a área de contato do pneu, com a finalidade de corrigir as possíveis distorções focais da máquina fotográfica e, por meio de uma câmara fotográfica digital, foram tiradas fotos de cada área de contato (Figura 3), utilizando-se de uma plataforma fixa e elevada na estrutura da respectiva prensa hidráulica.

Numa etapa final, as fotos digitais foram descarregadas em um microcomputador e, finalmente, as leituras gráficas dessas áreas de contato foram obtidas utilizando o software AUTOCAD, sendo as áreas ajustadas, empregando-se a relação expressa na eq.(1). 


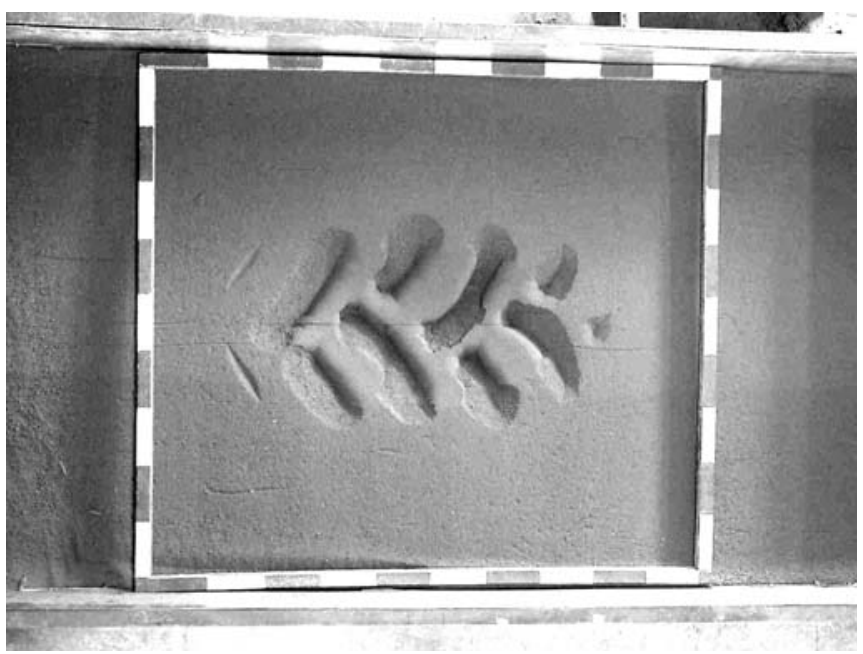

FIGURA 3. Área de contato.

$$
\mathrm{A}=\frac{\text { Aq Apf }}{\text { Aqf }}
$$

em que,

A - área de contato, $\mathrm{m}^{2}$

$\mathrm{A}_{\mathrm{q}}$ - área do quadro, $\mathrm{m}^{2}$;

$\mathrm{A}_{\mathrm{pf}}$ - área do pneu na foto, $\mathrm{m}^{2}$, e

$\mathrm{A}_{\mathrm{qf}}$ - área do quadro na foto, $\mathrm{m}^{2}$.

O recalque do pneu agrícola no solo consiste na profundidade de penetração do mesmo na amostra de solo, devido às cargas radiais aplicadas sobre o respectivo pneu. Para a avaliação dos recalques dos rodados pneumáticos, foi construído um perfilômetro que apresentava $0,95 \mathrm{~m}$ de largura, $1,95 \mathrm{~m}$ de comprimento e hastes com $0,38 \mathrm{~m}$ de comprimento. $\mathrm{O}$ perfilômetro apresentava 17 hastes em seu comprimento e $13 \mathrm{em}$ sua largura, distanciadas $0,15 \mathrm{~m}$, sendo o mesmo acoplado à estrutura do tanque de solo. O recalque foi obtido pela diferença de profundidade entre a haste situada no centro da área de contato e a externa à mesma.

As deformações totais consistem na somatória da deformação do pneu e do recalque do rodado pneumático no solo, devido à superfície deformável em que os pneus foram prensados, seguindo os procedimentos de carregamentos adotados, com as respectivas combinações de pressões de inflação para cada pneu.

Para a determinação das deformações totais, foi acoplada uma escala graduada na estrutura da prensa hidráulica. Avaliaram-se na escala graduada a dimensão referente ao contato inicial do pneu no solo e as deformações totais devido às cargas aplicadas ao eixo do rodado pneumático, essa última, obtida pela diferença com a primeira.

Devido à condição de superfície deformável em que os pneus foram prensados, obtiveram-se perfis característicos de deformações dos rodados pneumáticos sobre as amostras de solo, segundo os carregamentos aplicados sobre os pneus. As deformações elásticas dos pneus agrícolas foram determinadas por diferença entre as deformações totais e o recalque do pneu no solo.

Após cada prensagem do pneu sobre o solo, foi determinada a resistência do solo à penetração por um penetrógrafo manual SC-60. Avaliou-se a resistência do solo à penetração na área de contato e externa à mesma (testemunha), com a finalidade de obter-se o incremento da resistência do solo à 
penetração de cada pneu ao sofrer a aplicação das cargas radiais. Efetuaram-se cinco perfurações, longitudinalmente sobre as áreas de contato, distanciadas $0,17 \mathrm{~m}$; três perfurações transversalmente a uma distância de $0,15 \mathrm{~m}$ e três perfurações aleatórias externamente às áreas.

$\mathrm{O}$ experimento seguiu um delineamento casualizado, em esquema fatorial 3 x 4, com 12 tratamentos e quatro repetições, totalizando 48 observações. Os tratamentos foram formados pelas combinações de três tipos de pneus e quatro cargas radiais (lastragem).

Os dados obtidos foram submetidos à análise de variância, pelo teste $\mathrm{F}$, e à comparação de médias, pelo teste de Tukey, a 5\% de probabilidade.

\section{RESULTADOS E DISCUSSÃO}

A síntese da análise estatística é apresentada para grupos de variáveis na Tabela 1. Nessa Tabela, os dados referentes aos fatores pneu e carga radial representam médias de 16 e 12 observações, respectivamente, para áreas de contato, deformações elásticas dos pneus agrícolas e perfis de recalque dos pneus no solo. Para a variável incremento da resistência do solo à penetração, os dados referentes aos fatores pneu e carga radial representam médias de 112 e 84 observações, respectivamente.

TABELA 1. Síntese da análise de variância e do teste de médias para as variáveis área de contato, deformação elástica dos pneus agrícolas, perfil do recalque dos pneus no solo e incremento da resistência do solo à penetração.

\begin{tabular}{ccccc}
\hline Fatores & $\begin{array}{c}\text { Área de Contato } \\
\left(\mathrm{m}^{2}\right)\end{array}$ & $\begin{array}{c}\text { Deformação elástica } \\
\text { do pneu }(\mathrm{m})\end{array}$ & $\begin{array}{c}\text { Recalque do } \\
\text { pneu }(\mathrm{m})\end{array}$ & $\begin{array}{c}\text { Incremento da } \\
\text { Resistência do Solo à } \\
\text { Penetração }(\mathrm{kPa})\end{array}$ \\
\hline Pneu (P) & $0,257 \mathrm{a}$ & $0,0340 \mathrm{a}$ & $0,0515 \mathrm{~b}$ & $1274,17 \mathrm{~b}$ \\
BPAF & $0,223 \mathrm{~b}$ & $0,0275 \mathrm{~b}$ & $0,0624 \mathrm{a}$ & $1434,83 \mathrm{~b}$ \\
Radial & $0,186 \mathrm{c}$ & $0,0261 \mathrm{~b}$ & $0,0607 \mathrm{a}$ & $1743,81 \mathrm{a}$ \\
Diagonal & & & & \\
\hline Carga Radial $(\mathrm{C})$ & $0,169 \mathrm{c}$ & $0,0199 \mathrm{c}$ & $0,0443 \mathrm{~d}$ & $996,4 \mathrm{c}$ \\
$5 \mathrm{kN}$ & $0,212 \mathrm{~b}$ & $0,0241 \mathrm{c}$ & $0,0557 \mathrm{c}$ & $1506,6 \mathrm{~b}$ \\
$10 \mathrm{kN}$ & $0,240 \mathrm{a}$ & $0,0316 \mathrm{~b}$ & $0,0630 \mathrm{~b}$ & $1631,9 \mathrm{ab}$ \\
$15 \mathrm{kN}$ & $0,267 \mathrm{a}$ & $0,0413 \mathrm{a}$ & $0,0698 \mathrm{a}$ & $1802,2 \mathrm{a}$ \\
$20 \mathrm{kN}$ & & & & \\
Teste F & $30,89 *$ & $11,50 *$ & $32,31 *$ & $14,71 *$ \\
$\mathrm{P}$ & $32,20 *$ & $42,97 *$ & $84,99 *$ & $23,32 *$ \\
$\mathrm{C}$ & $4,64 *$ & $1,65 \mathrm{NS}$ & $1,65 \mathrm{NS}$ & $0,19 \mathrm{NS}$ \\
\hline PxC & 11,48 & 17 & 7,067 & 16,773 \\
\hline C.V. $\%$ & & & & \\
\hline
\end{tabular}

$\overline{\text { Em cada coluna, para cada fator, médias seguidas de mesma letra minúscula não diferem estatisticamente entre si, pelo }}$ teste de Tukey, a 5\% de probabilidade; NS - não significativo ( $P>0,05)$; * - significativo $(\mathrm{P}<0,01)$, e C.V. - coeficiente de variação.

\section{Área de contato de pneus agrícolas}

Pela Tabela 1, nota-se que o fator pneu influenciou significativamente no fator carga radial para a variável área de contato. $\mathrm{Na}$ Tabela 2, apresentam-se os dados dessa interação, e cada valor representa uma média de quatro observações.

Pela Tabela 2, observa-se que a variável área apresentou diferença significativa para todos os pneus ensaiados quando submetidos à carga de $15 \mathrm{kN}$, sendo que o valor da variável foi maior para o pneu BPAF, sofrendo um decréscimo para o pneu radial e menor para o pneu diagonal. A carga radial 
de $5 \mathrm{kN}$ não apresentou diferença significativa da variável área para todos os pneus ensaiados, e as cargas de $10 \mathrm{kN}$ e $20 \mathrm{kN}$ apresentaram valores da variável maior para o pneu BPAF, sofrendo decréscimo para os pneus radial e diagonal. Esse comportamento confere com SANTOS (2003) que observou maiores áreas de contato para o pneu BPAF.

TABELA 2. Interação entre os fatores pneu e carga $(\mathrm{kN})$ para a variável área de contato $\left(\mathrm{m}^{2}\right)$.

\begin{tabular}{lcccc}
\hline & \multicolumn{4}{c}{ Cargas $(\mathrm{kN})$} \\
\cline { 2 - 5 } Pneus & 5 & \multicolumn{4}{c}{10} & \multicolumn{1}{c}{15} \\
\cline { 2 - 5 } & $0,1598 \mathrm{a}$ & $0,2623 \mathrm{a}$ & $0,2933 \mathrm{a}$ & $0,3135 \mathrm{a}$ \\
BPAF & $0,1883 \mathrm{a}$ & $0,1968 \mathrm{~b}$ & $0,2458 \mathrm{~b}$ & $0,2620 \mathrm{~b}$ \\
Radial & $0,1600 \mathrm{a}$ & $0,1773 \mathrm{~b}$ & $0,1825 \mathrm{c}$ & $0,2255 \mathrm{~b}$ \\
Diagonal & &
\end{tabular}

Em cada coluna, para cada fator, médias seguidas de mesma letra minúscula não diferem estatisticamente entre si, pelo teste de Tukey, a 5\% de probabilidade.

Como houve interação significativa entre os fatores pneu e carga, como pode ser observado na Tabela 2, procurou-se ajustar regressões que melhor explicassem o comportamento das cargas para cada pneu ensaiado. Os resultados obtidos estão de acordo com o estudo realizado por ABEELS (1976), que define que a variação da área de contato de um pneu é diretamente proporcional à carga e inversamente proporcional à pressão de inflação. Na Figura 4, ilustram-se as regressões e suas equações, a 6,95\%; 6,44\% e 3,67\% de significância, para os pneus BPAF, diagonal e radial, respectivamente.
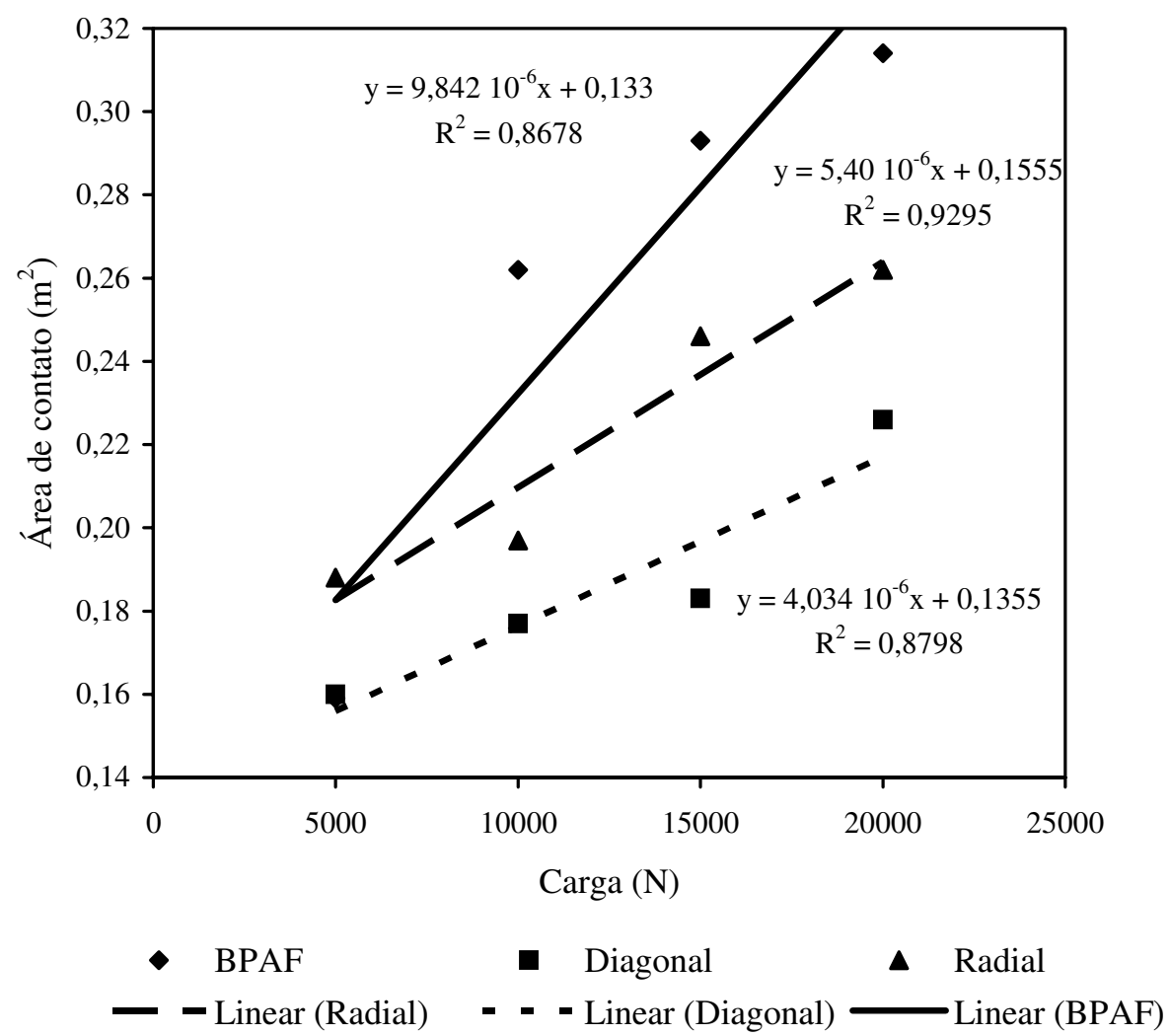

FIGURA 4. Regressão linear das áreas de contato em função das cargas para os pneus BPAF, diagonal e radial. 
Observando os coeficientes angulares das regressões da Figura 4, o pneu BPAF foi mais susceptível ao aumento de carga em termos de área de contato, seguido pelo radial e, por fim, o diagonal.

\section{Deformação elástica de pneus agrícolas}

Analisando-se a Tabela 1, observa-se que o pneu BPAF apresentou diferença significativa em relação aos outros pneus ensaiados para a variável deformação elástica, sendo que os pneus radiais e diagonais apresentaram menores valores de deformação elástica quando comparados com o pneu BPAF.

Pela Tabela 1, nota-se que a carga radial de 20 e $15 \mathrm{kN}$ apresentou diferença significativa em relação às outras cargas radiais para a variável deformação elástica, e a carga radial de $20 \mathrm{kN}$ apresentou valores superiores de deformação elástica em relação à carga de $15 \mathrm{kN}$, sofrendo um decréscimo para as cargas radiais de 10 e $5 \mathrm{kN}$.

\section{Perfil do recalque dos pneus no solo}

Analisando-se a Tabela 1, observa-se que o pneu BPAF apresentou diferença significativa em relação aos outros pneus ensaiados para a variável perfil do recalque do pneu no solo, e os pneus radiais e diagonais apresentaram maiores valores do perfil do recalque quando comparados com o pneu BPAF.

Pela Tabela 1, nota-se que a variável perfil do recalque do pneu no solo apresentou diferença significativa para todas as cargas radiais avaliadas, sendo que o valor da variável foi maior para a carga radial de $20 \mathrm{kN}$, sofrendo decréscimos conforme diminuíam as cargas radiais aplicadas sobre os pneus agrícolas.

\section{Incremento da resistência do solo à penetração}

Analisando-se a Tabela 1, observa-se que o pneu diagonal apresentou diferença significativa em relação aos outros pneus ensaiados para a variável incremento da resistência do solo à penetração, e os pneus BPAF e radial apresentaram menores valores de incremento da resistência do solo à penetração quando comparados com o pneu diagonal.

Pela Tabela 1, nota-se que a variável incremento da resistência do solo à penetração apresentou menor valor para a carga radial de $5 \mathrm{kN}$, diferindo estatisticamente das outras cargas. A carga radial de 10 e $15 \mathrm{kN}$ não diferiram estatisticamente para a variável incremento da resistência do solo à penetração, ocorrendo o mesmo ao analisar as cargas radiais de 15 e $20 \mathrm{kN}$.

\section{CONCLUSÕES}

A combinação entre tipo de pneu e cargas radiais mostrou maiores valores de área de contato para o pneu BPAF.

O pneu BPAF apresentou maior deformação elástica e menor perfil de recalque no solo, em comparação aos outros pneus ensaiados.

Os maiores incrementos da resistência do solo à penetração foram obtidos pelo pneu diagonal, reforçando a importância de utilizar os pneus BPAF e radial para culturas que apresentam alto tráfego agrícola.

A elevação gradativa das cargas radiais evidenciou um aumento das áreas de contato, das deformações elásticas, dos perfis de recalque dos pneus e da resistência do solo à penetração, independentemente do tipo de rodado pneumático. 


\section{AGRADECIMENTOS}

Ao NEMPA/FCA - UNESP e ao PIBIC/CNPq, que fomentaram este trabalho.

\section{REFERÊNCIAS}

ABEELS, P.R.J. Tire deflection and contact studies. Journal of Terramechanics, Oxford, v.13, n.3, p.183-96, 1976.

GOODYEAR. Farm tire handbook. Americana, 1994. 88 p.

MAZETTO, F.R. Avaliação das metodologias de determinação das áreas de contato e deformações elásticas de pneus agrícolas em função das pressões de inflação e cargas radiais. $2004.100 \mathrm{f}$. Dissertação (Mestrado em Energia na Agricultura) - Faculdade de Ciências Agronômicas, Universidade Estadual Paulista, Botucatu, 2004.

PORTERFIELD, J.W; CARPENTER, T.G. Soil compaction: an index of potential compaction for agricultural tires. Transactions of the ASAE, St. Joseph, v.29, n.4, p.917-22, 1986.

SANTOS, P. I. B. Método dos elementos finitos para determinação da área de contato, entre um rodado e uma superfície deformável. 2003. 203 f. Tese (Doutorado em Energia na Agricultura) Faculdade de Ciências Agronômicas, Universidade Estadual Paulista, Botucatu, 2003.

SHARMA, A.K; PANDEY, K.P. A review on contact area measurement of pneumatic tyre on rigid and deformable surfaces. Journal of Terramechanics, Oxford, v.33, n.5, p.253-64, 1996.

TRELLEBORG. Agricultural tyres manual. Trelleborg, 2002. 20 p.

UPADHYAYA, S.K.; WULFSOHN, D. Review of traction prediction equations. In: ASAE -

American Society of Agricultural Engineers, 12., 1990, St. Joseph. Anais... St. Joseph: ASAE, 1990. (Paper 90-1573). 